\title{
Treatment of Tumours and Tumour-like Lesions in the Foot and Ankle - A Single Institution Analysis
}

\author{
Kap Jung Kim, Sang Ki Lee, Yong Joo Chi, Shann Haw Chang, \\ Dae Geon Song and Hyun Jong Park \\ Department of Orthopaedic Surgery, Eulji University College of Medicine, \\ 1306 Dunsan-dong, Seo-gu, Daejeon(322-799), Republic of Korea \\ Correspondent Author: Kap-Jung Kim (E-mail: oskkj@eulji.ac.kr)
}

\begin{abstract}
We analyzed the results of tumours and tumour-like lesions arising in the foot and ankle and to establish the treatment guidelines. From 2006 to March 2013, 64 patients with 67 cases with primary tumours and tumour-like lesions arising in the foot and ankle were included. The benign lesions were 58 patients with 61 cases and the malignant tumours were 6 patients with 6 cases. Thirty patients were male and 34 patients were female. The mean age was 42.4 years old. The mean follow up period was 50.3 months. Fifty two lesions were located in the foot and 15 lesions were located in the ankle. Benign lesions were dominant, outnumbering malignant tumours by more than 10 to 1 . The most predominant benign lesion was ganglion. Treatment options for benign lesions were excisional biopsy in 57 cases, curettage/grafting in 3 cases, and amputation in 1 case. Treatment outcomes were continuously disease free in 55 patients and local recurrence in 3 patients. Regarding malignant tumours, wide resection was in 1 case and amputation in 5 cases. Treatment outcomes were continuously disease free in 3 cases and died of disease in 3 cases. Thorough surgical excision is important for treatment of benign tumours and tumour-like lesions for preventing local recurrence. Limb salvage surgery is difficult for malignant tumours due to the peculiar anatomic complexity of these regions. Lager scale studies of the foot and ankle tumours and tumour-like lesions are needed to understand this rare subgroup of neoplasm.
\end{abstract}

Key words: Foot, Ankle, Tumours, Tumour-like lesions

\section{Introduction}

The primary tumours and tumour-like lesions in the foot and ankle are rare in these locations [1-5]. Fewer than $2 \%$ of all sarcomas and fewer than $10 \%$ of soft tissue sarcomas arise in foot and ankle. Metastatic lesions are more uncommon. According to the rarity of these tumours, we are unaware of detailed clinicopathologic features, therapeutic approaches, and results in these locations. They do, however, occur with sufficient frequency to present a regular diagnostic challenge. Clinical manifestations are often non-specific, most commonly that of pain and soft tissue swelling. An inadequate or delayed diagnosis of tumours and tumour-like lesions in the foot and ankle has been correlated with greater rates of recurrence. Wide surgical resection and functional limb salvage are particularly difficult due to the 
anatomic complexity of the foot and ankle.

The purpose of our study is to analyze the clinicopathologic results of tumours and tumour-like lesions arising in the foot and ankle treated in a single institution retrospectively and to establish the guidelines for treatment in these locations.

\section{Materials and Methods}

The study was performed with the approval of the institutional review of board. Between 2006 and March 2013, 64 patients with 67 cases of primary musculoskeletal tumours and tumour-like lesions of foot and ankle were included in this study. A total 67 cases were identified, and data were obtained on age, gender, anatomic location, histologic type, stage, treatment option, and outcomes. Soft tissue sarcomas were staged using the American Joint Committee on Cancer staging system.[6] Lesions were histologically confirmed either benign or malignant, treated by single surgeon (K-J Kim) in single institution. Benign lesions were 58 patients with 61 cases $(91 \%)$ and malignant tumours were 6 patients with 6 cases $(9 \%)$. Thirty patients were male and thirty four patients were female. The mean age was 42.4 years old (range, 13 85). The mean follow up period was 50.3 months (range, 6 91). Fifty two lesions were located in the foot and 15 lesions were located in the ankle.

The present study did not include patients with pseudotumoral lesions, tumours treated with thermal ablation, or patients with metastatic lesions in the foot and ankle.

\section{Results}

\subsection{Benign bone and soft tissue lesions}

A total 58 patients with 61 benign lesions, 26 patients (45\%) were male and 32 patients (55\%) were female. The mean age was 40.5 years (range, 13 72). Forty six lesions (75\%) occurred in the foot; 19 cases (31\%) in forefoot, 22 cases (36\%) in midfoot and 5 cases (8\%) in hindfoot and 15 lesions (25\%) occurred in the ankle. The most predominant histologic diagnosis was ganglion, 22 cases $(36.2 \%)$ out of total 61 cases, which located tendon sheath in 12 cases (54.5\%) and joint capsule in 10 cases (45.5\%). Schwannoma and osteochondroma were the second predominant lesions in the foot and ankle, each tumour was 7 cases $(11.5 \%)$, respectively. Treatment options were excisional biopsy in 57 cases (93\%), curettage/grafting in 3 cases (5\%) and amputation in 1 case (2\%). One case of amputation was enchondroma occurred at distal phalanx of 2nd toe. Distal phalanx was enlarged with inner osteolytic lesion, tiny chondroid calcification, coarse trabeculation, distal cortical destruction, and combined with intra-articular fracture. Toe amputation was considered better than curettage and grafting.

Treatment outcomes were continuously disease free in 55 cases (95\%) and local recurrence in 3 cases (5\%). Recurred 3 cases were 2 cases of ganglion and 1 case of fibroma. Average disease-free survival period was 51.2 months (range, 6 91) (Table 1). 
Table 1. Demographic data of benign bone and soft tissue lesions in the foot and ankle

\begin{tabular}{|c|c|}
\hline Parameter & Value \\
\hline Mean age & $40.5 \mathrm{Y}$ (range, 13 72) \\
\hline \multicolumn{2}{|l|}{ Gender } \\
\hline Male & $26(45 \%)$ \\
\hline Female & $32(55 \%)$ \\
\hline \multicolumn{2}{|l|}{ Location } \\
\hline Foot & $46(75 \%)$ \\
\hline Forefoot & $19(31 \%)$ \\
\hline Midfoot & $22(36 \%)$ \\
\hline Hindfoot & $5(8 \%)$ \\
\hline Ankle & $15(25 \%)$ \\
\hline \multicolumn{2}{|l|}{ Histologic type } \\
\hline Ganglion & $22(36.2 \%)$ \\
\hline Schwannoma & $7(11.5 \%)$ \\
\hline Osteochondroma & $7(11.5 \%)$ \\
\hline Fibroma & $5(8.2 \%)$ \\
\hline Hemangioma & $5(8.2 \%)$ \\
\hline Enchondroma & $3(4.9 \%)$ \\
\hline PVNS & $3(4.9 \%)$ \\
\hline Xanthoma & $3(4.9 \%)$ \\
\hline Osteoid osteoma & $2(3.3 \%)$ \\
\hline Angiomyoma & $1(1.6 \%)$ \\
\hline Lipoma & $1(1.6 \%)$ \\
\hline Neuroma & $1(1.6 \%)$ \\
\hline Giant cell tumour & $1(1.6 \%)$ \\
\hline \multicolumn{2}{|l|}{ Treatment } \\
\hline Excisional biopsy & $57(93 \%)$ \\
\hline Curettage and grafting & $3(5 \%)$ \\
\hline Amputation & $1(2 \%)$ \\
\hline \multicolumn{2}{|l|}{ Outcome } \\
\hline Continuously disease free & $55(95 \%)$ \\
\hline Local recurrence & $3(5 \%)$ \\
\hline Average disease-free survival & $51.2 \mathrm{M}$ (range, 6 91) \\
\hline
\end{tabular}

Y=year; PVNS=Pigmented villo-nodular synovitis; $\mathrm{M}=$ months; Data presented as $n(\%)$, unless otherwise noted.

\subsection{Malignant bone and soft tissue lesions}

A total 6 patients with 6 malignant tumours, 4 patients $(67 \%)$ were male and 2 patients (33\%) were female. The mean age was 60.3 years (range, 49 85). The mean follow up period was 36.8 months (range, 11-60). All 6 malignant tumours (100\%) occurred in the foot; 2 cases $(33 \%)$ in forefoot, 2 cases $(33 \%)$ in midfoot and 2 cases $(33 \%)$ in hindfoot. None occurred in the ankle. The histologic types were malignant melanoma in 3 cases $(50 \%)$, squamous cell carcinoma in 2 cases (33\%) and Ewing's sarcoma in 1 case (17\%). Treatment options were wide resection in 1 case (17\%) and amputation in 5 cases (83\%). Treatment outcomes were continuously disease free in 3 cases (50\%) and died of disease in 3 cases $(50 \%)$. Average disease-free survival period was 36.8 months (range, 5 60) (Table 2). The Kaplan-Meier survival rate at postoperative 5 years was $50 \%$ (95\% confidence interval) (Figure 1). 
International Journal of Bio-Science and Bio-Technology

Vol.6, No.1 (2014)

Table 2. Demographic data of malignant bone and soft tissue lesions in the foot and ankle

\begin{tabular}{ll}
\hline Parameter & Value \\
\hline Mean age & $60.3 \mathrm{Y}$ (range, 49 85) \\
Gender & \\
Male & $4(67 \%)$ \\
Female & $2(33 \%)$ \\
Location & $6(100 \%)$ \\
Foot & $2(33 \%)$ \\
$\quad$ Forefoot & $2(33 \%)$ \\
$\quad$ Midfoot & $2(33 \%)$ \\
Hindfoot & 0 \\
Ankle & \\
Histologic type & $3(50 \%)$ \\
Malignant melanoma & $2(33 \%)$ \\
Squamous cell carcinoma & $1(17 \%)$ \\
Ewing's sarcoma & \\
Treatment & $1(17 \%)$ \\
Wide resection & $5(83 \%)$ \\
Amputation & \\
Stage & 6 \\
Soft tissue tumours & 0 \\
IA & 0 \\
IB & $3(50 \%)$ \\
IIA & $2(33 \%)$ \\
IIB & 0 \\
III & $1(17 \%)$ \\
IV & \\
Oncologic outcome & $3(50 \%)$ \\
Continuously disease free & $3(50 \%)$ \\
Died of disease & $41 \mathrm{M}(\mathrm{range}, 5 \sim 65)$ \\
Average disease-free survival & \\
\hline
\end{tabular}

Y=year; $\mathrm{M}=$ months; Data presented as $n(\%)$, unless otherwise noted.

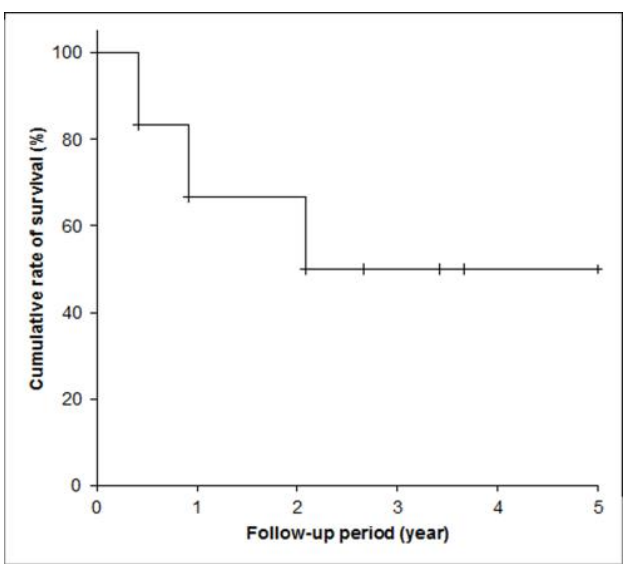

Figure 1. The Kaplan-Meier survival rate at postoperative 5 years was $50 \%$ (95\% confidence interval) 


\subsection{Complications}

Among benign bone and soft tissue lesions, 3 cases recurred as mentioned above; 2 cases of ganglion and 1 case of fibroma. Re-excision was performed in all recurred cases. Two cases of schwannoma remained mild numbness after surgery. These neurologic symptoms disappeared within postoperative 3 months and 5 months, respectively. No postoperative wound related complications occurred.

Regarding malignant bone and soft tissue tumours, superficial wound infection occurred in 1 case of Ewing's sarcoma. Postoperative wound infection was solved appropriate debridement. No other neurovascular compromise occurred.

\section{Discussion}

Like neoplasms at other sites, there is no specific clinical feature or characteristics specific for tumours in the foot and ankle. The most common clinical manifestations were pain, tenderness, edema, and a growing mass. The optimal treatment of a patient who has bone and soft tissue sarcoma begins with a proper work-up and a correctly performed biopsy that does not limit further definitive procedures. Because of rare incidence of foot and ankle tumorous lesions, the diagnosis is often made after the surgical excision of tumour that had been thought to be benign. The sex of the patient, a history of trauma, the duration of the symptoms, the presence of pain or neurological symptoms, and the size of the lesion were not useful discriminators between benign and malignant lesions. Thus, biopsy should be done before definitive surgery. Proper biopsy and accurate histologic confirm can prevent inadequate excision. Biopsy should be done only after clinical, laboratory, and radiographic examinations are complete. As stated previously, completion of the evaluation before biopsy aids in planning the placement of the biopsy incision, helps provide more information leading to a more accurate pathological diagnosis, and avoids artifacts on imaging studies. Adequate tissue should be obtained via biopsy. If the results of the evaluation suggest that a primary malignancy is in the differential diagnosis, the patient should be referred to a musculoskeletal oncologist before biopsy.

Ganglion is the most common benign soft tissue tumour-like lesion in the foot and ankle [7]. Treatment of options of ganglion are aspiration, rupture the cyst, injection of sclerosing agent, and surgery. Treatment principle of ganglion in our institution is doing nothing at early stage of diagnosis. We rarely do aspiration or rupture the cyst which leads to frequent recurrence. If the ganglia develop in the foot, they are often symptomatic because of their larger average size relative to wrist ganglia $[8,9]$. Surgical excision is done only when symptom develops or recurrence. Pain and mass effect associated with cyst can make wearing shoes difficult and uncomfortable. Ahn et al reported that 36 cases (67.9\%) of ganglion in the foot and ankle had pain as the main reason of operation in their study [10]. We experienced that a 58-year-old female patient complained of ovoid mass occurring at dorsum of the left foot and foot pain while wearing shoes. Plain radiograph of foot showed oval shaped soft tissue shadow suggestive of soft tissue mass arising from dorsum of the foot. T2-weighted sagittal scan of foot MRI revealed oval shaped ganglionic cyst occurred at 2nd metatarsal-cuneiform joint level around EDL tendon. On operation, main mass originated from EDL tendon sheath with attaching to the periosteum. Complete excision of the mass was done with special attention (Figure 2). The reported recurrence rate of ganglion after excision is $5 \%$ to $30 \%[11,12]$. We experienced 2 cases $(9.1 \%)$ of recurred ganglion out of total 22 cases. The main reason of recurrence was the failure of identification and removal of the satellite mass might have led to the recurrence. Careful preoperative evaluation and meticulous surgical technique can help lower the recurrence rate. 


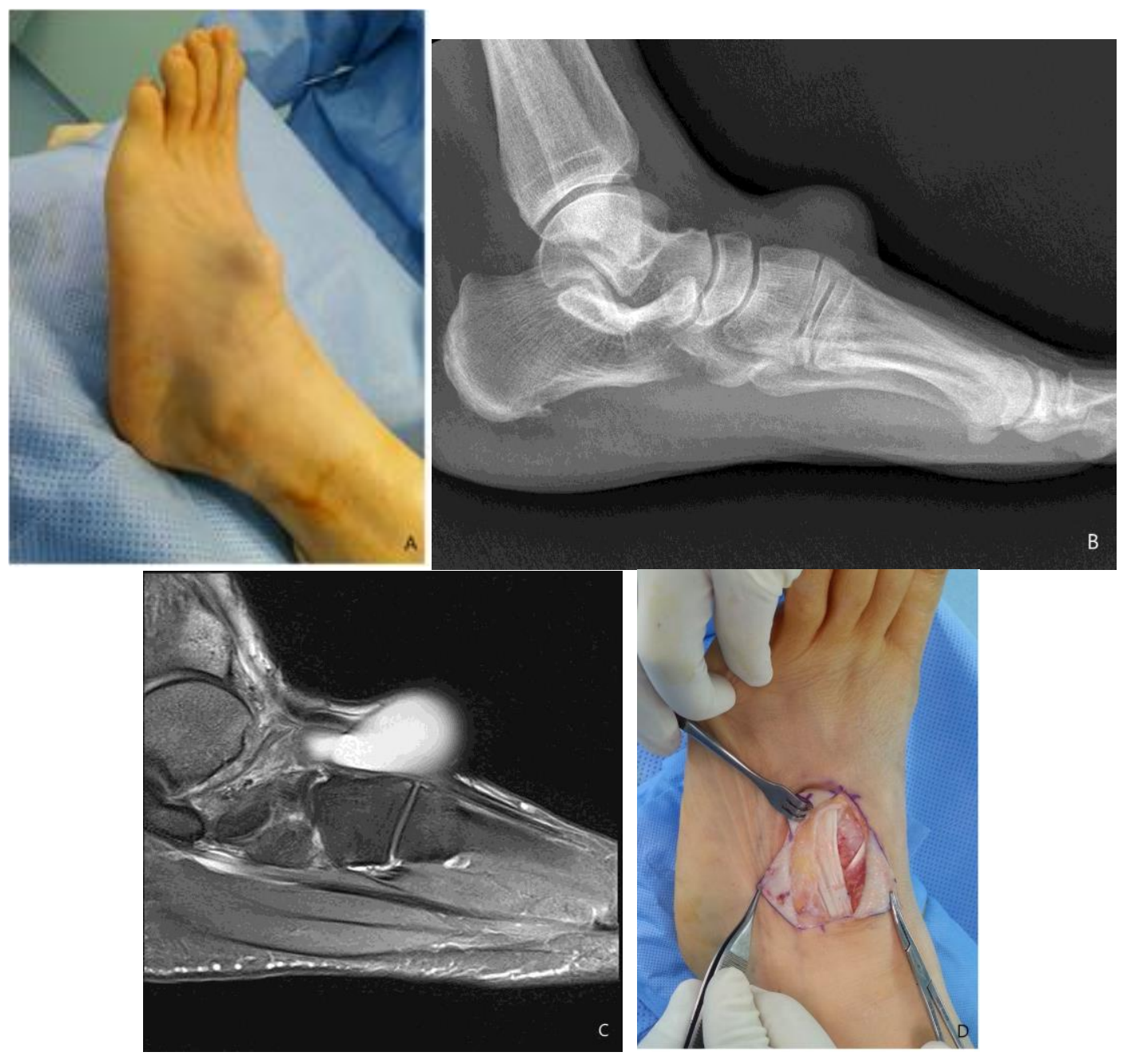

Figure 2. A 58-year-old female complained of ovoid mass occurring at dorsum of the foot. (A) Photograph showing the oval shaped mass at dorsum of the foot. (B) Plain radiograph showing the oval shaped soft tissue shadow arising from dorsum of the foot. (C) T2-weighted sagittal scan of foot MRI revealed oval shaped ganglionic cyst occurred at 2nd metatarsal-cuneiform joint level around EDL tendon. (D) Intraoperative photograph showing main mass originated from EDL tendon sheath with attaching to the periosteum.

Ozger et al., reported 16 cases of malignant tumours of the foot and ankle from 1989 to 1998 [13]. They found male predominance, a median age of 33 years, a more frequent tumour location in the foot, and a soft tissue tumour rate of $81 \%$. These results were quite similar to ours. We experienced 6 cases of primary malignant tumours in the foot and ankle. Male versus female was 4:2. All 6 cases occurred in the foot. All cases were soft tissue origin malignant tumours.

Below knee amputation has been the standard surgical procedure for malignant bone and soft tissue tumour in the foot and ankle $[14,15]$. In the foot, the usual barriers to tumour growth are less well developed than in more proximal parts of the extremity. The cortex of the midfoot and hindfoot is thin and is perforated by numerous vascular channels. The compact structure of the foot and ankle leads to early tumour involvement of adjacent bone 
and soft tissue tissues [16]. Most of patients had extracompartmental tumor invasion at the time of initial diagnosis. Furthermore, involvement of the neurovascular structure and anantomical complexity of foot and ankle make limb salvage surgery difficult for malignant bone and soft tissue tumour in the foot and ankle. We experienced that a 45 -year-old male patient complained of rapid growing huge foot mass. Mass had developed within 6 months. T2-weighted sagittal scan of foot MRI revealed multilobular septated huge soft tissue mass with involving cortical erosion of cuneiform and the base of 1st metatarsal bone. Limb salvage was thought to be impossible. Thus, below knee amputation was done (Figure 3). At the time of diagnosis, the patient had multiple pulmonary metastases. The patient has survived for 5 months after amputation. Histologic diagnosis was Ewing's sarcoma.

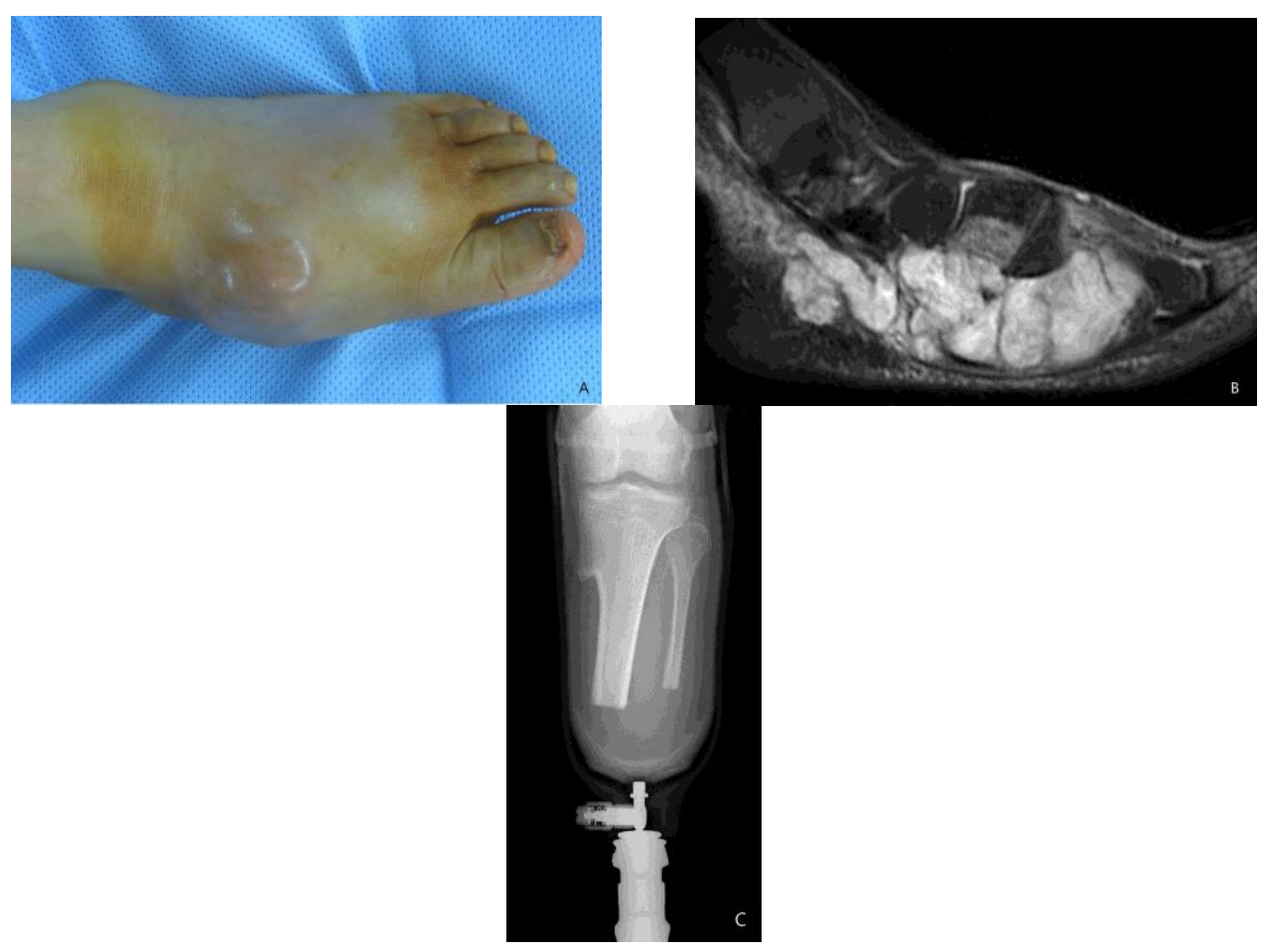

Figure 3. A 45-year-old male patient complained of rapid growing huge foot mass. (A) Photograph showing round and huge mass developed medial side of foot. (B) T2-weighted sagittal scan of foot MRI revealed multilobular septated huge soft tissue mass with involving cortical erosion of cuneiform and the base of 1st metatarsal bone. (C) Plain radiograph showing below knee amputation

Sometimes, benign looking round soft tissue mass revealed malignant soft tissue sarcoma. We experienced another interesting case mimicking simple heel mass. A 58-year-old patient complained of right heel pain. The pain was aggravated when walking or running. Plain radiograph seemed normal foot. Foot MRI was taken for further evaluation. T1-weighted sagittal scan of foot MRI revealed round heel mass well-located in heel pad with homogeneous signal. We regarded as benign cystic mass and simple marginal excision was done. However, histologic report was surprisingly different. It was confirmed malignant melanoma of heel. Wide resection with previous incision scar and subsequent sural flap was done for covering heel defect (Figure 4). 


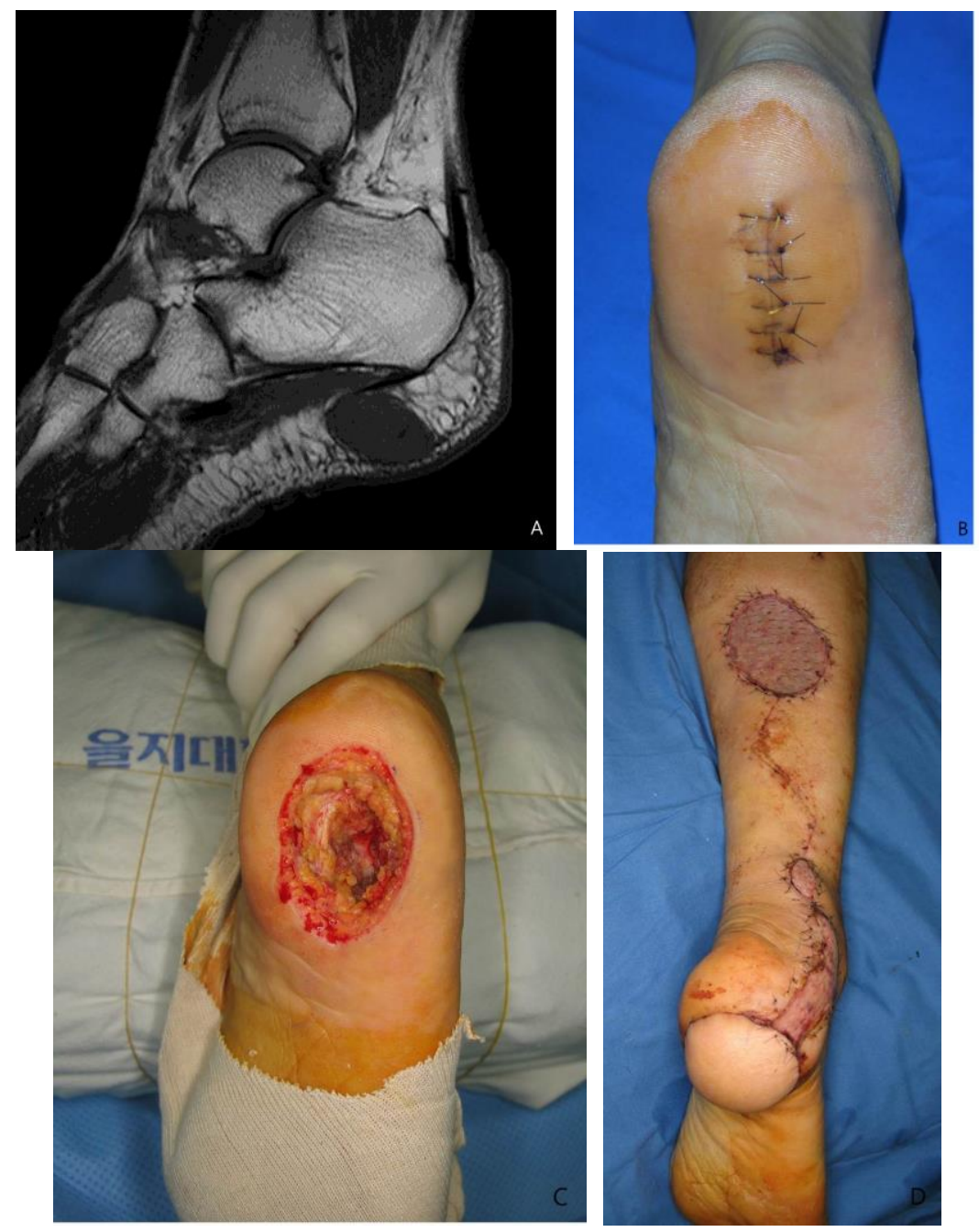

Figure 4. A 58-year-old male patient complained of right heel pain. (A) T1weighted sagittal scan of foot MRI revealed round mass with homogeneous signal. (B) Photograph showing simple excision. (C) Photograph showing wide resection for obtaining wide and safe margin. (D) Photograph showing concomitant sural flap for covering heel defect

\section{Conclusion}

In conclusion, tumours and tumour-like lesions of the foot and ankle are rare and are associated with a peculiar clinical presentation, histologic diagnoses, surgical and medical approach, and patient outcome. The adequate diagnosis and proper treatment have a significant effect on the patient's outcome. In our series of 64 patients with 67 tumours and tumour-like lesions, the vast majority were located in the foot. Benign lesions were dominant, outnumbering malignant more than 10 to 1 . The diversity of histologic benign types was evident. The major limitation of our study for malignant bone and soft tissue tumours in the foot and ankle was weak statistical power due to small number of cases. We didn't check functional outcome such as AOFAS score.[17] Larger scale studies of patients with tumours 
of the foot and ankle are needed to understand this rare subgroup of neoplasms.

\section{Acknowledgements}

No benefits in any form have been received or will be received from a commercial party related directly or indirectly to the subject of this article.

\section{References}

[1] R. Biscaglia, A. Gasbarrini, T. Böhling, P. Bacchini, F. Bertoni and P. Picci, "Osteosarcoma of the bones of the foot-an easily misdiagnosed malignant tumor", Mayo Clin Proc., vol. 73, (1998), pp. 842-847.

[2] L. B. Chou and M. M. Malawer, "Analysis of surgical treatment of 33 foot and ankle tumors", Foot Ankle Int., vol. 15, (1994), pp. 175-181.

[3] R. J. Gray, B. A. Pockaj, M. L. Vega, S. M. Connolly, D. J. DiCaudo, T. A. Kile and E. W. Buchel, "Diagnosis and treatment of malignant melanoma of the foot", Foot Ankle Int., vol. 27, (2006), pp. 696-705.

[4] E. J. Kirby, M. J. Shereff and M. M. Lewis, "Soft-tissue tumors and tumor-like lesions of the foot. An analysis of eighty-three cases.”, J Bone Joint Surg Am., vol. 71, (1989), pp. 621-626.

[5] C. P. Azevedo, J. M. Casanova, M. G. Guerra, A. L. Santos, M. I. Portela and P. F. Tavares, "Tumors of the foot and ankle: a single-institution experience", J Foot Ankle Surg., vol. 52, (2013), pp. 147-152.

[6] S. B. Edge, D. R. Byrd and C. C. Compton, "AJCC Cancer Staging Manual", 7th ed. New York: Springer, (2010), pp. 291.

[7] E. J. Kirby, M. J. Shereff and M. M. Lewis, "Soft-tissue tumors and tumor-like lesions of the foot. An analysis of eighty-three cases”, J Bone Joint Surg Am., vol. 71, (1989), pp. 621-626.

[8] A. Ogose, T. Hotta, H. Kawashima and N. Endo, "A painful large ganglion cyst of the ankle treated by the injection of OK-432”, Mod Rheumatol., vol. 17, (2007), pp. 341-343.

[9] R. Ortega, D. P. Fessell, J. A. Jacobson, J. Lin, M. T. Van Holsbeeck and C. W. Hayes, "Sonography of ankle ganglia with pathologic correlation in 10 pediatric and adult patients", AJR Am J Roentgenol., vol. 178, (2002), pp. 1445-1449.

[10] J. H. Ahn, W. S. Choy and H. Y. Kim, "Operative treatment for ganglion cysts of the foot and ankle", J Foot Ankle Surg., vol. 49, (2010), pp 442-445.

[11] A. C. Angelides and P. F. Wallace, "The dorsal ganglion of the wrist: its pathogenesis, gross and microscopic anatomy, and surgical treatment", J Hand Surg Am., vol. 1, (1976), pp. 228-235.

[12] B. V. McEvedy, "Simple ganglia”, Br J Surg., vol. 49, (1962), pp. 585-594.

[13] H. Ozger, L. Eralp, M. Türker and M. Basaran, "Surgical treatment of malignant tumor of the foot and ankle", Int J Clin Oncol., vol. 10, (2005), pp. 127-132.

[14] T. Ozaki, A. Hillmann, N. Linder and W. Winkelmann, "Surgical treatment of bone sarcomas of the fibula. Analysis of 19 cases", Arch Orthop Trauma Surg., vol. 116, (1997), pp. 475-479.

[15] M. T. Selch, K. H. Kopald, G. A. Ferreiro, J. M. Mirra, R. G. Parker and F. R. Eilber, "Limb salvage therapy for soft tissue sarcomas of the foot", Int J Radiat Oncon Biol Phys., vol. 19, (1990), pp. 41-48.

[16] W. F. Enneking, "Musculoskeletal tumor surgery”, Churchill-Livingstone, New York, (1983), pp. 720-726.

[17] H. B. Kitaoka, I. J. Alexander, R. S. Adelaar, J. A. Nunley, M. S. Myerson and M. Sanders, "Clinical rating systems for the ankle-hindfoot, midfoot, hallux, and lesser toes”, Foot Ankle Int., vol. 15, (1994), pp. 349-353. 
International Journal of Bio-Science and Bio-Technology Vol.6, No.1 (2014) 